\title{
Varying Speed of Light, Modified Chaplygin Gas and Accelerating Universe
}

\author{
Anup Kumar Singha ${ }^{1}$ and Ujjal Debnath ${ }^{2 *}$ \\ ${ }^{1}$ Department of Mathematics, Calcutta Institute of Technology, Uluberia-711 316, India. \\ ${ }^{2}$ Department of Mathematics, Bengal Engineering and Science University, Shibpur, Howrah-711 103, India.
}

(Dated: July 27, 2018)

\begin{abstract}
In this paper, we have considered a model of modified Chaplygin gas in VSL theory with variable gravitational constant $G$. We have shown that the evolution of the universe starts from radiation era to phantom model. The whole evolution of the universe has been shown diagramatically by using statefinder parameters.

PACS numbers: $98.80 . \mathrm{Cq}, 95.35 .+\mathrm{d}$
\end{abstract}

\section{INTRODUCTION}

Recently, the matter field can give rise to an accelerated expansion [1] for the universe stems from the observational data regarding the luminosity-redshift relation of type Ia supernovae $[2,3]$ up to about $z \sim 1$. This matter field is called quintessence matter (shortly Q-matter). This Q-matter can behave like a cosmological constant $[4,5]$ by combining positive energy density and negative pressure. So there must be this Q-matter either neglected or unknown responsible for this accelerated universe. At the present epoch, a lot of works has been done to solve this quintessence problem and most popular candidates for Q-matter has so far been a scalar field having a potential which generates a sufficient negative pressure. The Q-matter behaves as a perfect fluid with a barotropic equation of state and so some effort has been investigated in determining its adiabatic index at the present epoch [6,7]. Chimento et al [8] have showen that a combination of dissipative effects such as a bulk viscous stress and a quintessence scalar field gives an accelerated expansion for an open universe $(k=-1)$. Banerjee et al [9] also have shown that it is possible to have an accelerated universe with BD-theory in Friedmann model without any matter.

Another alternative candidate for Q-matter is exotic type of fluid - the so-called Chaplygin gas which obeys the equation of state $p=-B / \rho,(B>0)[10]$, where $p$ and $\rho$ are respectively the pressure and energy density. Subsequently, the above equation was generalized to the form $p=-B / \rho^{\alpha}, 0 \leq \alpha \leq 1[11,12]$ and recently it was modified to the form $p=A \rho-B / \rho^{\alpha},(A>0)[13,14]$, which is known as Modified Chaplygin Gas. This model represents the evolution of the universe starting from the radiation era to the $\Lambda C D M$ model.

The possibility that the speed of light $c$ might vary has recently attracted considerable attention. In a cosmological setting, the variations in $c$ have been shown to solve the cosmological puzzles - the horizon, flatness and Lambda problems of big-bang cosmology. The variations of velocity of light can also solve the quasi-lambda problems. For power-law variations in the velocity of light with the cosmological scale factors, Barrow et al [15] have shown that flatness problem can be solved. The Machian VSL scenario in which $c=c_{0} a^{n}$, introduced by Barrow [16] has significant advantages to the phase transition scenario in which the speed of light changes suddenly from $c^{-}$to $c^{+}$, preferred by Albrecht and Magueijo [17]. For changing $c$, the geometry of the universe is not affected. In classical electromagnetism the speed of light is only constant in vacuum and it 'varies' in dielectric media. The changing $c$ breaks Lorentz invariance and conservation of energy. The geometry of Universe is not affected by a changing $c$. One can allowed a changing $c$ to do the job normally done by superluminal expansion. For changing $c$, the gravitational laws should be modified. The basic assumption is that a variable $c$ does not induce corrections to curvature in the cosmological frame and therefore, Einstein's equations, relating curvature to stress energy are still valid. The reason behind this postulate is that $c$ changes in the Local Lorentzian frames associated with cosmological expansion. The effect is a special relativistic effect and not a gravitational effect. Therefore curvature should not be related with the variation of $c$.

The organization of the paper is as follows: In section II, we have studied field equations and its solutions

*ujjaldebnath@yahoo.com , ujjal@iucaa.ernet.in 
due to VSL theory for modified Chaplygin gas with varying $G$. In section III, we have discussed whole stage of the evolution of the universe using statefinder parameters. In the last section (i.e., section IV) we give some remarks of this paper.

\section{BASIC EQUATIONS AND SOLUTIONS}

In VSL theory and varying $G$, the Einstein equations for FRW model is

$$
\frac{\dot{a}^{2}}{a^{2}}+\frac{k c^{2}(t)}{a^{2}}=\frac{8 \pi G(t) \rho}{3}
$$

and

$$
\frac{\ddot{a}}{a}=-\frac{4 \pi G(t)}{3}\left(\rho+\frac{3 p}{c^{2}(t)}\right)
$$

where $p$ and $\rho$ are the pressure and energy density respectively. Here we consider modified Chaplygin gas in VSL theory as

$$
p=A \rho c^{2}(t)-\frac{B}{\left\{\rho c^{2}(t)\right\}^{\alpha}} \quad, A>0, \quad B>0, \quad 0 \leq \alpha \leq 1
$$

The energy conservation equation incorporating time variation in $c(t)$ and $G(t)$ is

$$
\dot{\rho}+3 \frac{\dot{a}}{a}\left(\rho+\frac{p}{c^{2}}\right)=-\frac{\dot{G}}{G} \rho+\frac{3 k c \dot{c}}{4 \pi G a^{2}}
$$

Now assume, the velocity of light and $G$ are in power-law form of scale factor as

$$
c(t)=c_{0} a^{n}
$$

and

$$
G=G_{0} a^{m}
$$

where $c_{0}$ and $G_{0}$ are both positive constants. Since we know that speed of light decreases with time and $G$ increases with time, so that $n$ must be negative and $m$ must be positive.

Using equations (3), (4), (5) and (6) we have the solution of $\rho$ as (for $k=0$ )

$$
\rho=\left[M a^{-2 n(1+\alpha)}+N a^{-(m+3 A+3)(1+\alpha)}\right]^{\frac{1}{1+\alpha}}
$$

where $M=\frac{3 B}{(3+3 A-2 n+m) c_{0}^{2(1+\alpha)}}, N=\frac{c_{1}}{g_{0}^{1+\alpha}}$ and $c_{1}$ is a constant.

Putting the value of $\rho$ from (7) in (2), we have

$$
\int \frac{a^{\frac{3 A+1}{2}} d a}{\left[M a^{(3-2 n+m+3 A)(1+\alpha)}+N\right]^{\frac{1}{2(1+\alpha)}}}=c_{2} t
$$

where $c_{2}=\sqrt{\frac{8 \pi G_{0}}{3}}$.

The solution of scale factor $a(t)$ has the form 
$a^{\frac{3(1+A)}{2}}{ }_{2} F_{1}\left[\frac{1}{2(1+\alpha)}, \frac{3(1+A)}{2(3+3 A+m-2 n)}, 1+\frac{3(1+A)}{2(3+3 A+m-2 n)},-\frac{M}{N} a^{3+3 A+m-2 n}\right]=\frac{3}{2}(1+A) c_{2} N^{\frac{1}{2(1+\alpha)}} t$

where ${ }_{2} F_{1}$ is the hypergeometric function.

Now for small value of scale factor $a(t)$ i.e., for early universe, $\rho$ is very large and corresponds to the universe dominated by an equation of state $p=A \rho c^{2}$.

Also for large value of scale factor $a(t)$ i.e., for late universe, the relation between $p$ and $\rho$ satisfies the equation of state

$$
p=-\left(1+\frac{m-2 n}{3}\right) \rho c^{2}
$$

So evolution of the universe starts from radiation era $(A=1 / 3)$ to phantom model. From observations, we can conclude that $m \leq 2(n+1)$. For $\Lambda C D M$ model we have $m=2 n$.

\section{STATEFINDER PARAMETERS AND EVOLUTION OF THE UNIVERSE}

In 2003, V. Sahni et al [18] proposed a pair of parameters $\{r, s\}$, called statefinder parameters. In fact, trajectories in the $\{r, s\}$ plane corresponding to different cosmological models demonstrate qualitatively different behaviour. The above statefinder diagnostic pair has the following form:

$$
r=\frac{\dddot{a}}{a H^{3}} \quad \text { and } \quad s=\frac{r-1}{3\left(q-\frac{1}{2}\right)}
$$

where $H\left(=\frac{\ddot{a}}{a}\right)$ and $q\left(=-\frac{a \ddot{a}}{\dot{a}^{2}}\right)$ are the Hubble parameter and the deceleration parameter respectively. These parameters allow us to characterize the properties of dark energy. Trajectories in the $\{r, s\}$ plane corresponding to different cosmological models, for example $\Lambda$ CDM model diagrams correspond to the fixed point $s=0, r=1$.

The expressions for $q, r$ and $s$ are in the following:

$$
\begin{gathered}
q=\frac{1+3 A}{2}-\frac{3 B}{2\left(\rho c^{2}\right)^{1+\alpha}} \\
r=-\frac{1+3 A}{2}+\frac{3 B}{2\left(\rho c^{2}\right)^{1+\alpha}}-\frac{3}{2} m G_{0} a^{m}\left(1+A c^{2}-\frac{B}{\left(\rho c^{2}\right)^{1+\alpha}}\right) \\
-\frac{a}{2}\left(1+3 A+\frac{3 \alpha B}{\left(\rho c^{2}\right)^{1+\alpha}}\right) \frac{d \rho}{d a}+3 n\left(A \rho-\frac{B}{\rho^{\alpha} c^{2 \alpha+2}}\right)
\end{gathered}
$$

and

$$
\begin{gathered}
s=\frac{1}{3\left(A-\frac{B}{\left(\rho c^{2}\right)^{\alpha}}\right)}\left[-(1+A)+\frac{B}{\left(\rho c^{2}\right)^{1+\alpha}}-m G_{0} a^{m}\left(1+A c^{2}-\frac{B}{\left(\rho c^{2}\right)^{1+\alpha}}\right)\right. \\
\left.-\frac{a}{3}\left(1+3 A+\frac{3 \alpha B}{\left(\rho c^{2}\right)^{1+\alpha}}\right) \frac{d \rho}{d a}+2 n\left(A \rho-\frac{B}{\rho^{\alpha} c^{2 \alpha+2}}\right)\right]
\end{gathered}
$$
form.

The expressions of $r$ and $s$ are very complicated, so we can not found the relation between $r$ and $s$ in closed 


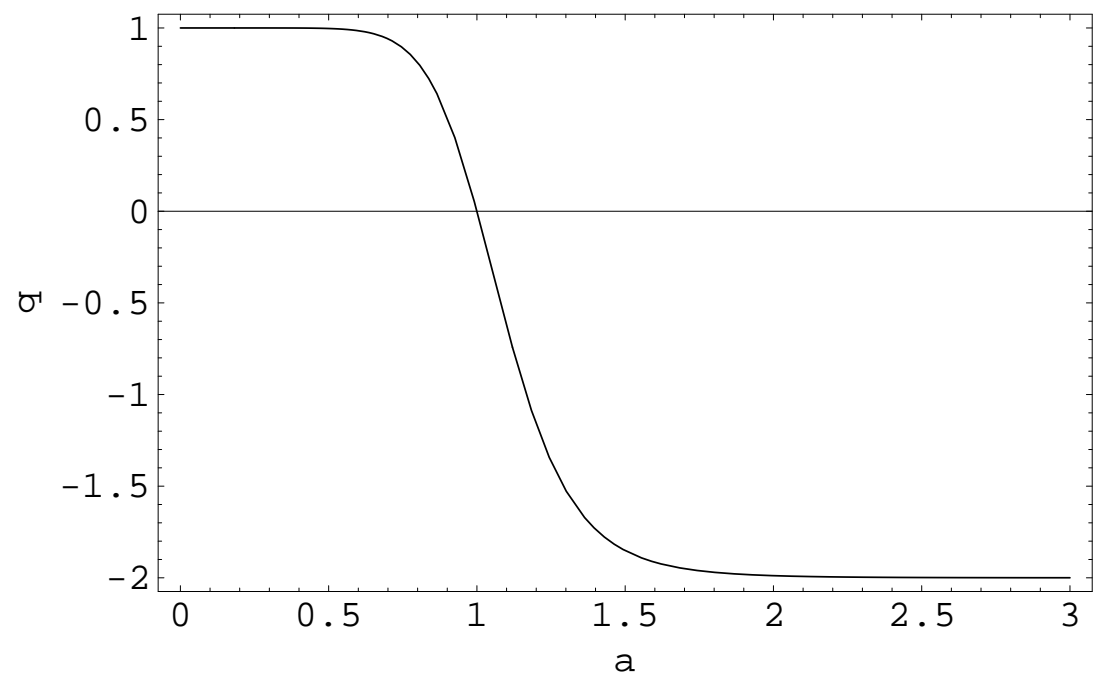

Fig.1

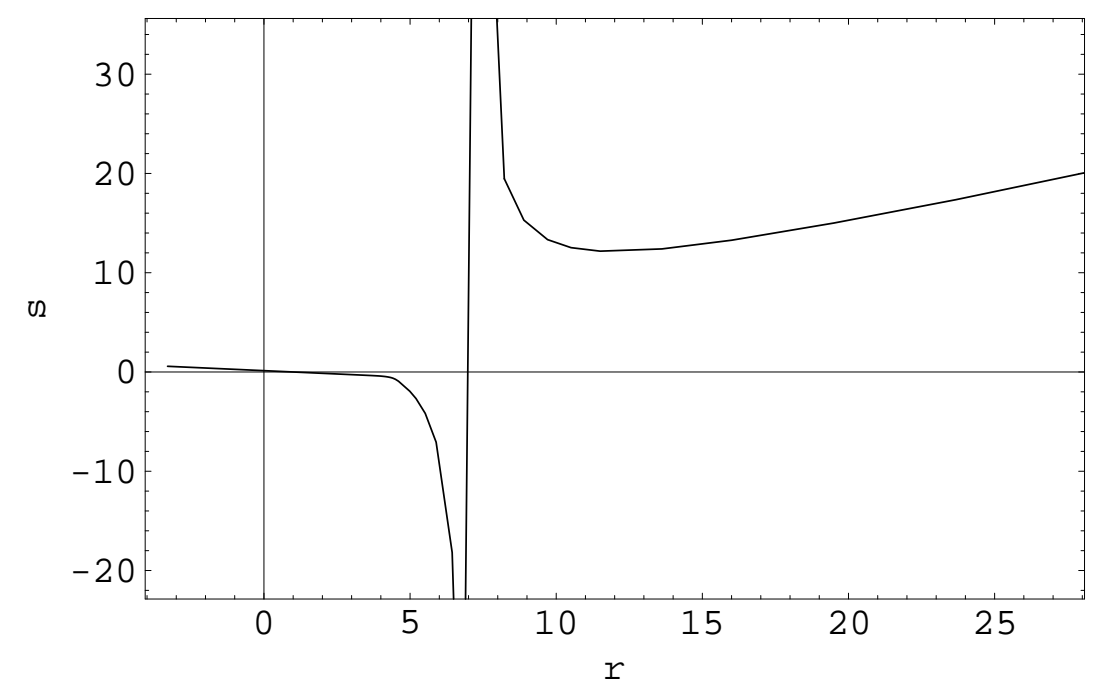

Fig.2

Fig.1 shows variation of $q$ against $a$ and Fig.2 shows variation of $s$ against $r$ for $\alpha=1 / 2, A=1 / 3, B=1, n=-1 / 2, m=1$.

Fig. 1 shows the variation of $q$ against $a$ for $A=1 / 3, \alpha=1 / 2, B=1, n=-1 / 2, m=1$. The plot of $q$ against $a$ reveals that the deceleration parameter indeed has a sign flip in the desired direction and indicates an early deceleration $(q>0)$ followed by a late time acceleration $(q<0)$ of the universe. From fig. 2 we see that the curve starts from the radiation era and goes asymptotically to he dust model for $s>0$ and $r>7$. But for $r<7$, the portion of the curve represents the evolution from dust state $(s=-\infty)$ to phantom model $(s>0)$ and this curve crosses $\Lambda \mathrm{CDM}$ stage $(r=1, s=0)$. Thus the total curve represents the evolution of the universe starting from radiation era to phantom model. 


\section{DISCUSSIONS}

In this work, we have proposed a model for modified Chaplygin gas in VSL theory with variable $G$. In this model, we have found the evolution of the universe starts from radiation era to phantom model. If $m=n=0$ i.e., $c=$ constant and $G=$ constant then we can recover our previous results [14]. Thus variable $c$ and $G$ can drive the evolution of the universe from radiation to phantom stages instead of $\Lambda$ CDM stage. From equation (10) we see that for $\Lambda \mathrm{CDM}$ model $m=2 n$ and for phantom model $m \leq 2(n+1)$. If $G=$ constant or $c=$ constant then any one of variable $G$ and $c$ can drive the evolution of the universe into the phantom model. Thus variable $G$ or $c$ has significant role for the evolution of the universe in large stages.

\section{References:}

[1] N. A. Bachall, J. P. Ostriker, S. Perlmutter, P. J. Steinhardt, Science 284 (1999) 1481.

[2] S. Perlmutter et al 1998 Nature (London) 391 51; 1999 Astrophys J 517565.

[3] A. G. Riess et al 1998 Astrophys J 116 1009; P. M. Garnavich et al 1998 Astrophys J 50974.

[4] R. R. Caldwell, R. Dave and P. J. Steinhardt, Phys. Rev. Lett. 80, 1582 (1998) [astro-ph/9708069].

[5] V. Sahni, A. A. Strarobinsky, Int. J. Mod. Phys. D 9373 (2000).

[6] L. Wang, R. Caldwell, J. P. Ostriker and P. J. Steinhardt, Astrophys. J. 53017 (2000).

[7] I. Waga and P. M. R. Miceli, Phys. Rev. D 59103507 (1999).

[8] L. P. Chimento, A. S. Jakubi and D. Pavo'n, Phys. Rev. D 62063508 (2000) (Preprint astro-ph/005070).

[9] N. Banerjee and D. Pavo'n, Phys. Rev. D 63043504 (2000).

[10] A. Kamenshchik, U. Moschella and V. Pasquier, Phys. Lett. B 511265 (2001).

[11] V. Gorini, A. Kamenshchik and U. Moschella, Phys. Rev. D 67063509 (2003); U. Alam, V. Sahni, T. D. Saini and A.A. Starobinsky, Mon. Not. Roy. Astron. Soc. 344, 1057 (2003).

[12] M. C. Bento, O. Bertolami and A. A. Sen, Phys. Rev. D 66043507 (2002).

[13] H. B. Benaoum, hep-th/0205140.

[14] U. Debnath, A. Banerjee and S. Chakraborty, Class. Quantum Grav. 215609 (2004).

[15] J. D. Barrow and J. Magueijo, Phys. Lett. B 443104 (1998); Phys. Lett. B bf 447246 (1999); Class. Quantum Grav. 161435 (1999).

[16] J. D. Barrow, Phys. Rev. D 59043515 (1999).

[17] A. Albrecht and J. Magueijo, Phys. Rev. D 59043516 (1999).

[18] V. Sahni, T. D. Saini, A. A. Strarobinsky and U. Alam, JETP Lett. 77201 (2003). 